\title{
Electron and Positron Density Distribution of Bloch Waves
}

\author{
G. LeHMPFUHL \\ Fritz-Haber-Institut der Max-Plandk-Gesellschaft, Berlin-Dahlem * \\ (Z. Naturforsch. 28a, 1-8 [1973]; received 24 October 1972) \\ Dedicated to Professor Dr. G. Borrmann on his 65th birthday
}

\begin{abstract}
The charge density distribution in the strongest Bloch waves for a dynamical many-beam diffraction situation was calculated for electrons and positrons. Near the [110] zone axis of $\mathrm{MgO}$ there exist three strong Bloch waves for electrons. One Bloch wave is concentrated at the rows of $\mathrm{Mg}$-atoms, a second at the rows of $\mathrm{O}$-atoms and a third one between the atoms. The positron Bloch waves are mainly concentrated between the atom rows and have only small charge density at the positions of the atoms. For an incident beam parallel to the [110] axis there exists only one strong positron Bloch wave while for electrons more than three Bloch waves are strong, explaining the channeling behaviour of positrons and electrons. Strong partial waves of different electron Bloch waves can be identified in the diffraction pattern from a $\mathrm{MgO}$ crystal wedge.
\end{abstract}

In a previous paper ${ }^{1}$ it was shown that for an exact description of the diffraction situation in a single crystal the interaction of many waves has to be taken into account. That means that the situation in the crystal must be described by many Bloch waves, these are wave fields, consisting of many plane waves. The Bloch waves describe the three-dimensional density distribution of the steady state of the electrons, travelling through the crystal.

The diffraction situation is well understood in the two-beam approximation because of the analytical formulation. In the two-beam case there exist two wave fields or Bloch waves each consisting of two partials waves. The electron density associated with one wave field has maxima in the planes of the atoms while the other wave field has the maxima between these planes as was shown first by NIEHRs ${ }^{2}$ and lateron e. g. by Hashimoto, Howie and Whelan ${ }^{3}$. One has to consider each wave field as an observable quantity. The two wave fields suffer different inelastic interactions with the lattice, leading to different $a b-$ sorption coefficients. This Borrmann effect for electrons or anomalous absorption effect was firstly observed by Honjo and Mrhama ${ }^{4}$ and lateron investi-

Reprint requests to Dr. G. Lehmpfuhl, Fritz-HaberInstitut der Max-Plandk-Gesellschaft, D-1000 Berlin 33, Faradayweg 4-6.

* Abteilung Prof. Dr. K. Molière.

1 G. Lehmpfuhl, Z. Naturforsch. 27a, 425 [1972].

2 H. Niehrs, Proc. Stodkholm Conference on Electron Microsc. 1956.

3 H. Hashimoto, A. Howie and M. J. Whelan, Proc. Roy. Soc. London A 269, 80 [1962].

4 G. Honjo and K. Minama, J. Phys. Soc. Japan 9, 184 [1954]. gated by different authors in electron diffraction as well as in electron microscopy $3,5,6$.

By the diffraction from a crystal wedge the Bloch waves are separated into their partial waves and the strongest ones can be observed and analysed separately. The diffraction spots show a fine-structure where each fine-structure spot represents a strong partial wave of a particular Bloch wave ${ }^{7}$. It is possible to find different partial waves of one Bloch wave in different reflections. They all belong 'o one branch of the dispersion surface.

Because of the absorption of the electrons the finestructure spots are elongated in a direction perpendicular to the projection of the edge onto the screen. The resulting intensity distribution in the fine-structure spot is the Fourier transform of the amplitude distribution of the Bloch wave at the exit surface. Due to a theoretical treatment of NIEHRS ${ }^{8}$ one can directly measure the absorption coefficient of the wave field from the halfwidth of the fine-structure spot. So it is possible to measure the inelastic interaction of the electrons with the crystal in different wave fields, especially in well-defined many-beam situations.

The differences of the absorption processes can be

5 H. Watanabe, A. Fukuhara, K. Kohra, J. Phys. Soc. Japan 17, Suppl. B-II, 195 [1962]. - G. MEYER-EHMSEN, Z. Physik 218, 352 [1969]. - P. Goodman and G. Lehmpfuhl, Acta Cryst. 22, 14 [1967].

6 G. Lehmpfuhl and K. Molière, J. Phys. Soc. Japan 17, Suppl. B-II, 130 [1962].

7 G. Lehmpfuhl and K. Molière, Z. Physik 166, 389 [1961].

8 H. Niehrs, Phys. Verh. 5, 175 [1954]. 
shown very clearly by looking at the electron density distribution of the wave fields. Such calculations for many-beam interactions were done by MeTherell and SPRING ${ }^{9}$ and by GJønNes and HøIER ${ }^{10}$ showing the different density distributions in a projection of the unit cell. Similar calculations of the field strength distribution for an X-ray three-beam case were done by HILDEBRANDT ${ }^{11}$ explaining the anomalous absorption.

\section{Bloch waves in $\mathrm{MgO}$}

The calculations of the electron density distribution become most interesting for the analysis of electron diffraction experiments with a crystal wedge. For a direction of the incident electron beam near the lowindexed zone axis [110] of $\mathrm{MgO}$ one observes many wave fields consisting of many strong partial waves ${ }^{7}$. The Bloch waves are displayed into their partial waves and the strongest ones can be analysed directly. In this orientation there exist lattice planes containing only oxygen atoms or magnesium atoms. In Fig. 1a we see
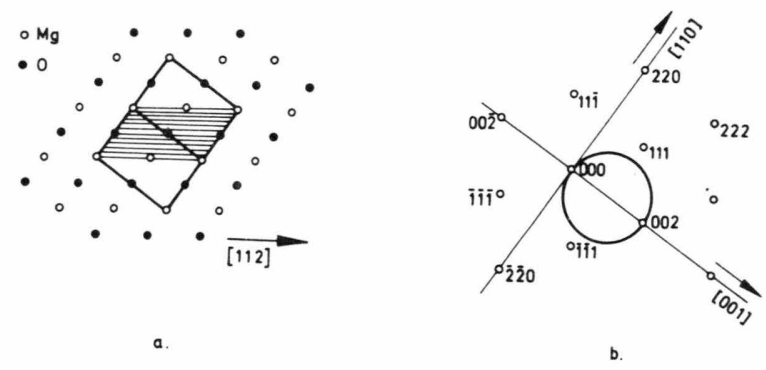

Fig. 1. (a) Projection of the atoms of a MgO crystal on the (110) plane. The full and open circles indicate rows of atoms. The projection of the primitive cube standing on one edge is indicated.

(b) The corresponding reciprocal lattice plane (110) with the intersection circle of the Ewald sphere. The direction of the incident beam is determined by the section points of the Ewald sphere with the [110] axis called $G$ and with the [001] axis called $H$. In the figure the orientation $G=0$ and $H=2$ is shown.

the projection of the atoms of the $\mathrm{MgO}$ lattice onto the (110) plane. The open circles indicate the $\mathrm{Mg}$ atoms and the full circles the oxygen-atoms. Each circle represents a row of atoms projected onto the (110) plane. The face centered unit cell is indicated, standing on one edge of the cube.

9 A. J. F. Metherell and M. S. Spring, VII. Intern. Congr. on Electr. Microsc., Grenoble B I, 105 [1970]. 10 J. Gjønnes and R. Høier, Acta Cryst. A 27, 166 [1971].
The electron density distribution in the strongest wave fields was calculated for a direction of the incident beam lying in the (110) plane with 002 exactly excited. The situation in the reciprocal space is shown in Fig. 1b where the intersection circle of the Ewald sphere with the reciprocal (110) plane is indicated. The wave field $\psi_{\mathrm{i}}(\boldsymbol{r})$ is given by

$$
\psi_{\mathrm{i}}(\boldsymbol{r})=\sum_{g} \psi_{g^{(\mathrm{i})}} \exp \left(2 \pi i \boldsymbol{K}_{g}(\mathrm{i}) \cdot \boldsymbol{r}\right) .
$$

$\psi_{g}{ }^{(1)}$ is the amplitude of the partial wave of wave field (i) belonging to the interference $g$ ( $g$ stands for the triple index $g_{1} g_{2} g_{3}$ of a vector $\boldsymbol{g}$ in the reciprocal lattice), $\boldsymbol{K}_{g}{ }^{(1)}=\boldsymbol{K}_{o}^{(1)}+\boldsymbol{g}$ is the crystal wave vector of the partial wave $\psi_{g}$ (i) and $\boldsymbol{r}$ a vector in the real space. The crystal wave vector $\boldsymbol{K}_{0}{ }^{(1)}$ is determined from the dynamical fundamental equation in the form $\boldsymbol{K}_{0}^{(1)}=1 / \lambda$ $\left(\boldsymbol{S}_{\mathrm{e}}+\boldsymbol{N}_{\mathrm{e}} \cdot \tau_{\mathrm{i}}\right)$ with $\boldsymbol{S}_{\mathrm{e}}$ a unit vector in the direction of the incident beam, $\boldsymbol{N}_{\mathrm{e}}$ a unit vector perpendicular to the entrance surface and $\tau_{\mathrm{i}}$ the "Anpassung" due to Bethe, determined from the dispersion equation (e. g. Ref. ${ }^{12}$ ). The wave fields are numbered in the order of the branches of the dispersion surface beginning from the top.

The electron density distribution $D(\boldsymbol{r})$ was calculated instead of the electron current density distribution because of the small differences of the crystal wave vectors $\boldsymbol{K}_{g}{ }^{(1)}$ for medium energy electrons $(50 \mathrm{keV})$

$$
D(\boldsymbol{r})=\psi_{\mathrm{i}}(\boldsymbol{r}) \cdot \psi_{\mathrm{i}}^{*}(\boldsymbol{r}) .
$$

If all vectors $\boldsymbol{g}$ of the reciprocal lattice lie in the (110) plane the electron density distribution is the same in all planes parallel to this plane. That means for this particular direction of incidence only reflections from the zero Laue zone have been taken into account.

The electron density distribution in three strong wave fields is shown in Figure 2. The calculation was done by a 42-beam approximation for the orientation shown in Figure 1b. The electron density was calculated in the (110) plane of the real lattice along the [112] direction indicated in Fig. 1a within the hatched area. The sequence of sections gives a three dimensional impression of the electron density distribution which is naturally not true in this form. The rows of atoms projected in the (110) plane are indicated by open and full circles. Wave field 1 (Fig. 2a) has maxima of den-

11 G. Hildebrandt, Phys. Stat. Sol. 24, 245 [1967].

12 G. Lehmpfuhl and A. Reissland, Z. Naturforsch. 23a, 544 [1968]. 
sity at the positions of the projected magnesium-atoms in the (110) plane. Wave field 2 (Fig. 2b) has maxima at the oxygen-positions and wave field 3 (Fig. 2c) has the maxima between the atoms. In the diffraction pattern

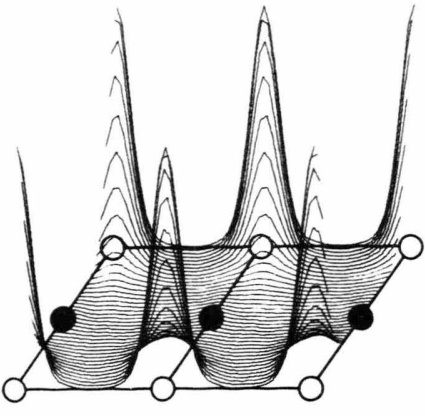

a)

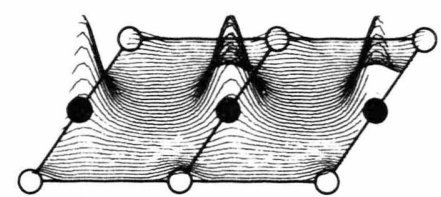

b)

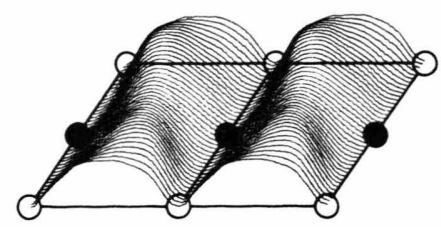

c)

Fig. 2. $(a-c)$ Electron density distribution in the strongest Bloch waves for $G=0$ and $H=2$. The atoms projected in the (110) plane are indicated by open circles $(\mathrm{Mg})$ and full circles $(\mathrm{O})$ (42-beam approximation, 50keV).

one observes for this direction of incidence three strong fine-structure spots of the 002 interference, that means three strong partial waves of three different wave fields. The fine-structure can be seen very clearly in a section through the dispersion surface ${ }^{12}$ recorded in the 002 interference in Figure 3a. The arrow indicates the orientation of the crystal for which the calculation was done. At this orientation the three strong branches of the dispersion surfaces have a minimum distance ${ }^{1}$. The scale $G$ indicates the intersection of the Ewald sphere with the [110] axis of the reciprocal lattice.

By comparing the experimentally observed diffraction pattern with the many-beam calculation one can associate the fine-structure spot or the branch of the dispersion surface with the corresponding wave field. This gives the possibility of investigating separately the interaction of the electrons with individual kinds of atoms of the lattice. Electrons of branch 1 at $G=$ 0 in Fig. 3a have mainly suffered interactions with the $\mathrm{Mg}$-atoms, while the electrons of branch 2 have suffered interactions with the oxygen-atoms and the electrons of branch 3 travel through the crystal between the atom rows.

The different branches of the dispersion surface or the different fine-structure spots of the diffraction pattern can also be associated with different Bloch waves by a simple model. The electrons are accelerated when entering the crystal. They see different potentials of the crystal in different Bloch waves. The electrons concentrated at the rows of $\mathrm{Mg}$-atoms belonging to Bloch wave 1 in Fig. $2 a$ are more accelerated into the crystal than the electrons of other Bloch waves. So the electrons of the uppermost branch of the dispersion surface in Fig. 3a must belong to Bloch wave 1 . The electrons of Bloch wave 2 concentrated at
(1)

(2)

(3)

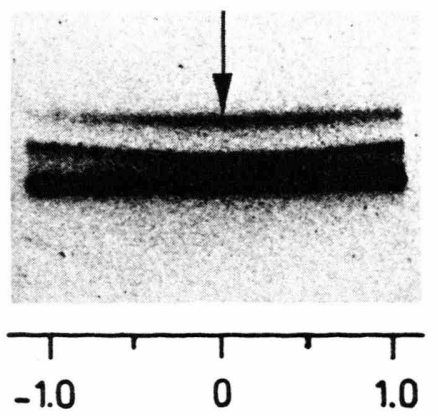

a)
(1)

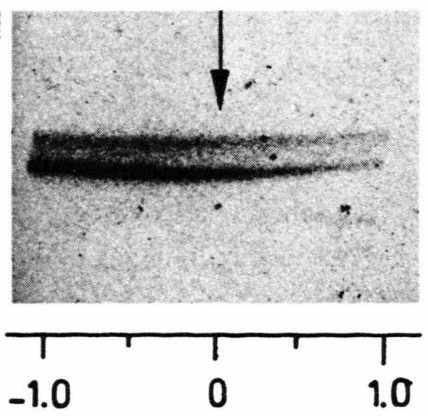

b)

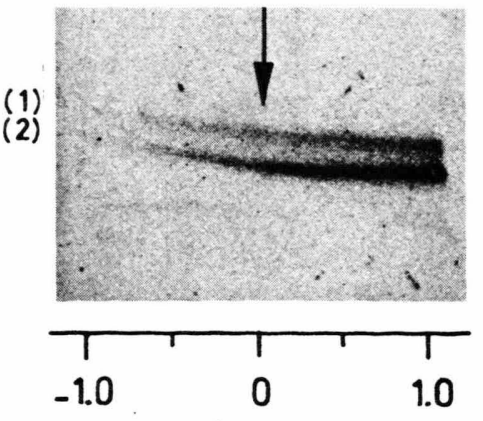

c)

Fig. 3. Section through the dispersion surface recorded in 002 (a), in 111 (b) and in $\overline{1} 11$ (c) with $H=2$. The scale indicates $G$, the section of the Ewald sphere with the [110] axis, e.g. $G=2$ means, that the Ewald sphere goes through the reciprocal lattice point 220. 
the rows of oxygen-atoms are less accelerated and one can find them in branch 2 of the dispersion surface. The electrons of Bloch wave 3 travelling between the rows of atoms have suffered the smallest acceleration. They belong to branch 3 of the dispersion surface.

A similar behaviour one finds for positrons which are discussed in the last paragraph. Positrons are decolerated when they enter the crystal. The positrons travell ing between the atoms have suffered the smallest deceleration. They belong to Bloch wave 1 and $c$ ne finds them in the uppermost branch of the dispersiun surface. As is easily seen the other Bloch waves behave correspondingly.

\section{Absorption}

The different interaction of the Bloch waves with the crystal can easily be seen by the different broadening of the branches of the dispersion surface due to the different absorption coefficients of the wave fields. Each wave field (i) will be absorbed with increasing distance $\xi$ from the entrance surface with an individual absorption coefficient $\mu_{\mathrm{i}}$ due to relation (3)

$$
\psi_{\mathrm{i}}(\boldsymbol{r}) \cdot \exp \left(-\mu_{\mathrm{i}} \xi\right)
$$

That means that each partial wave $\psi_{g}{ }^{(1)}$ of this wave field is absorbed with the same absorption coefficient. By this decrease of the amplitudes with increasing distance from the edge, the fine-structure spots are broadened. The absorption coefficients can be determined experimentally by measuring the halfwidth of the branch or by measuring the integrated intensity of the partial wave ${ }^{6}$. These are two independent methods. The halfwidth $H_{\mathrm{i}}$ of a fine-structure spot belonging to wave field (i) is proportional to the absorption coefficient of this wave field

$$
H_{\mathrm{i}} \propto \mu_{\mathrm{i}}
$$

The integrated intensity however is proportional to the reciprocal absorption coefficient multiplied by the intensity of the partial wave calculated from a manybeam approximation without absorption

$$
I_{l, \mathrm{nt}}^{(1)} \propto\left|\psi_{(1)}^{(1)}\right|^{\mathbf{2}} \cdot \mu_{\mathrm{i}}^{-1}
$$

The analysis of the fine-structure profile and especially the measurement of the halfwidth is the accurate method of determining the absorption coefficient. The measurement of the integrated intensity gives only an additional test.

Figure $4 \mathrm{a}$ shows the photometer-record of the intensity profile of the dispersion surface at $G=0$ in Figure $3 a$. It is difficult to measure the profile of branch 2 be-

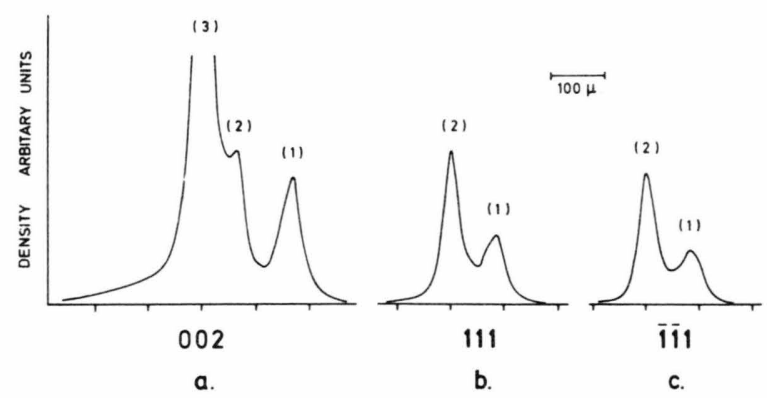

Fig. 4. $(a-c)$ Photometer-record across the sections through the dispersions surface in Fig. 3 at $G=0$ indicated by the arrows.

cause of the strong influence of branch 3 . The dispersion surface however can be recorded simultaneously in other reflections as e. $\mathrm{g}$. in 111 and $\overline{1} \overline{1}$, shown in Figure $3 \mathrm{~b}$ and $\mathrm{c}$. Here we see only the two branches 1 and 2. Branch 3 is very weak because of crystal symmetry as described in detail by Tinnappel ${ }^{13}$. The photometer-records of these sections through the dispersion surface are shown in Figure $4 \mathrm{~b}$ and $\mathrm{c}$. From these curves it is easy to determine the absorption coefficient of wave field 2 , that means the absorption coefficient due to the interaction with the oxygen-atoms. Wave field 3 contains the information of the interaction with the charge distribution between the atoms. This wave field is the strongest one, having the smallest absorption coefficient. This shows that the electrons of this wave field can travel with least interaction through the crystal. Branch 1 of the dispersion surface shows the largest broadening as we see in Fig. 3 corresponding to the strong absorption of the electrons of wave field 1 at rows of the $\mathrm{Mg}$-atoms.

The separations of the branches recorded in 002 and 111 are different because of coherent overlapping of the fine-structure spots ${ }^{14}$. The influence of this effect on structure-potential determination and measuring of the absorption coefficient was discussed briefly at

14 R. Didszunn, Thesis, FU Berlin 1969. 
the Kyoto Conference ${ }^{15}$ and will be published in detail shortly ${ }^{16}$.

The importance of considering a sufficient number of beams to describe the diffraction situation in the crystal can be demonstrated by an 8-beam calculation. In Fig. 5 we see the electron density distribution in the 3 wave fields. By the 8-beam calculation one cannot describe the sharp concentration of the electron den-

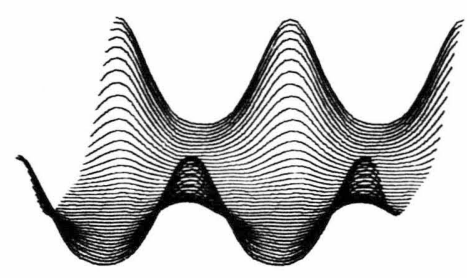

a)

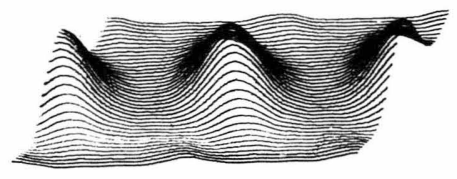

b)

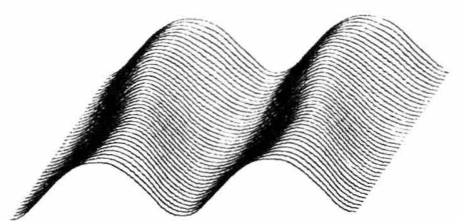

c)

Fig. 5. $(a-c)$ Electron density distribution in the three strongest Bloch waves for $G=0$ and $H=2$ calculated by an 8-beam approximation.

sity at the positions of the atoms. This shows very clearly that one has to consider the interaction of many beams for an accurate description of the scattering of electrons by a single crystal.

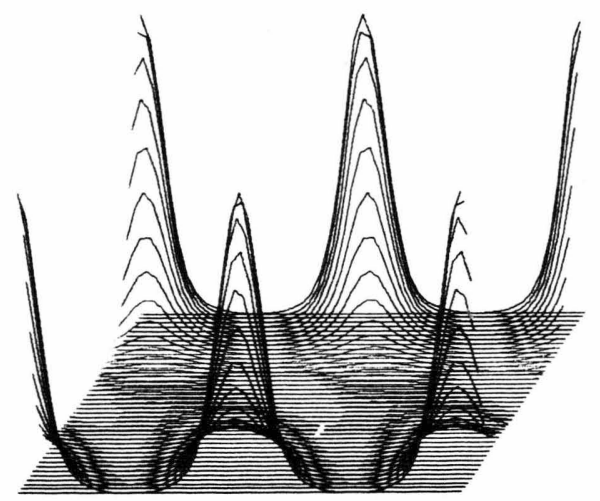

a)

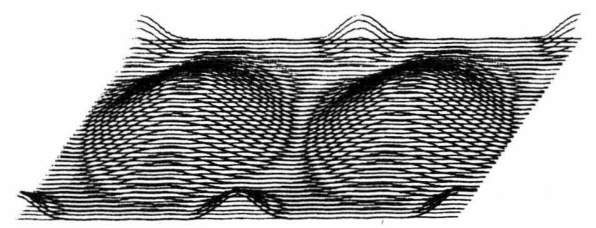

c)

\section{Orientation}

The density distribution of the electrons travelling through the crystal depends strongly on the orienta-

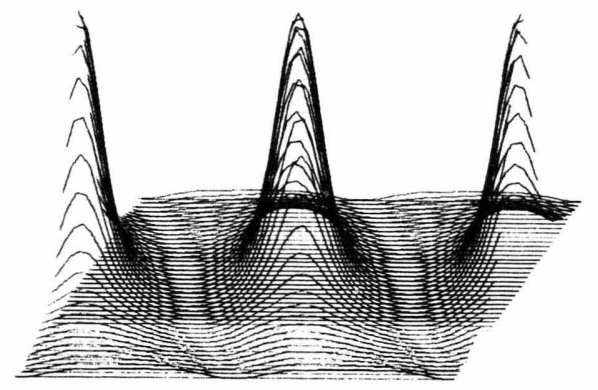

b)

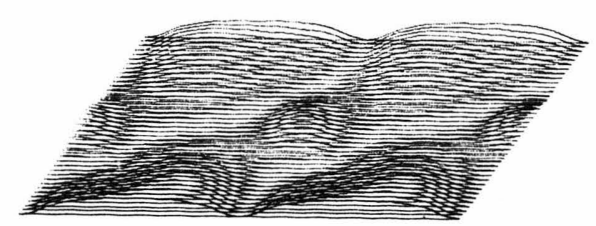

d)

Fig. 6. (a-d) Electron density distribution of the strongest Bloch waves for $G=1$ and $H=1$. The horizontal lines produce a moiré effect indicating regions of equal density.

15 A. W. S. Johnson and G. Lehmpfuhl, IX. Intern. Congr. of Crystallogr., Kyoto 1972.
$16 \mathrm{~K}$. Ishida, A. W. S. Johnson and G. Lehmpfuhl, to be published in Z. Naturforsch. 
tion of the crystal. For slightly different orientations the density distributions are shown in Figures 6 and 7. $G$ and $H$ indicate the intersection points of the Ewald sphere with the [110] and with the [001] axes of the reciprocal lattice (see caption of Fig. 1). For $G=1$ and $H=1$ the 111-interference is excited. For this orientation wave field 2 has strong maxima at the positions of the oxygen-atoms, similar to the maxima of wave field 1 at the rows of the $\mathrm{Mg}$-atoms. Beside wave field
3 and 4 there exist 6 weak wave fields which are just visible at that scale. The horizontal lines in the density distribution of Fig. 6 indicate by the Moiré-effect regions of equal density.

A completely different electron density distribution shows Fig. 7 in 5 wave fields for an orientation $G=2$ and $H=2$ where 002,220 and 222 are simultaneously excited. The inelastic interaction of the electrons with different parts of the unit cell can be investigated by

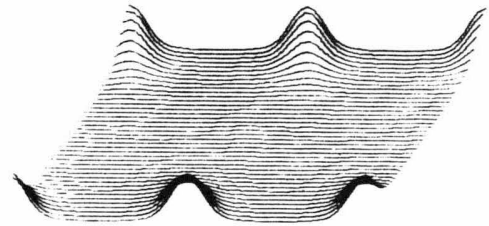

a)

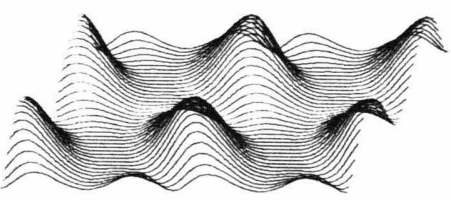

d)

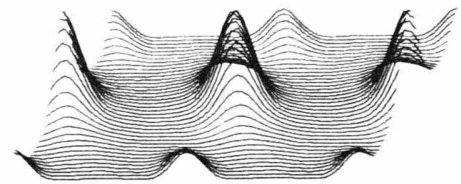

b)

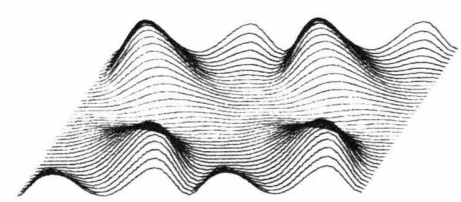

e)

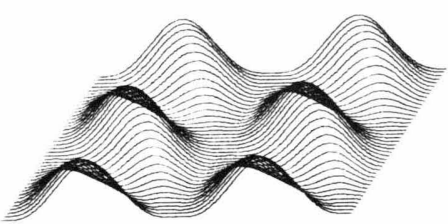

c)

Fig. 7. (a-e) Electron density distribution in the strongest Bloch waves for $G=2$ and $H=2$.

measuring the absorption coefficients of these wave fields from a fine-structure profile analysis as mentioned above.

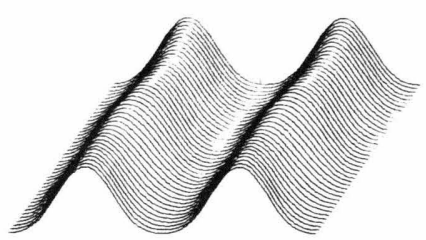

a)

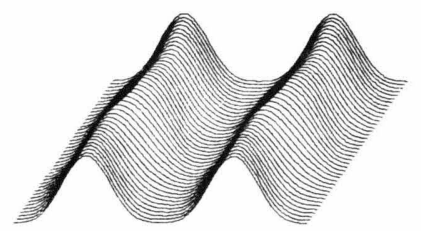

d)

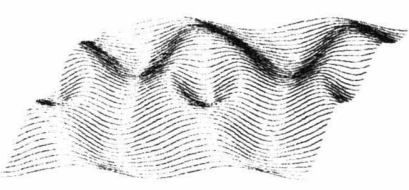

b)

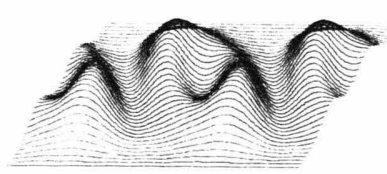

e)

\section{Channeling}

The strong concentration of the electron density in the Bloch waves along the rows of atoms near a low-

Fig. 8. (a-c) Positron density distribution in the Bloch waves for $50 \mathrm{keV}$ positrons at $G=0$ and $H=2$. (d-f) Bloch waves for $500 \mathrm{keV}$ positrons. 
indexed zone axis indicates the possibility of understanding electron channeling by the Bloch wave representation. In the particle picture of electron channeling one assumes the electrons travelling along the rows of positively charged nuclei. This picture is in agreement with the wave mechanical treatment.

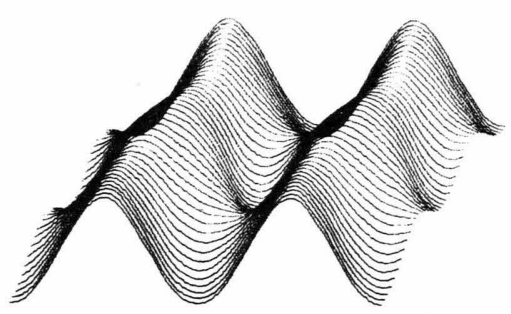

a)

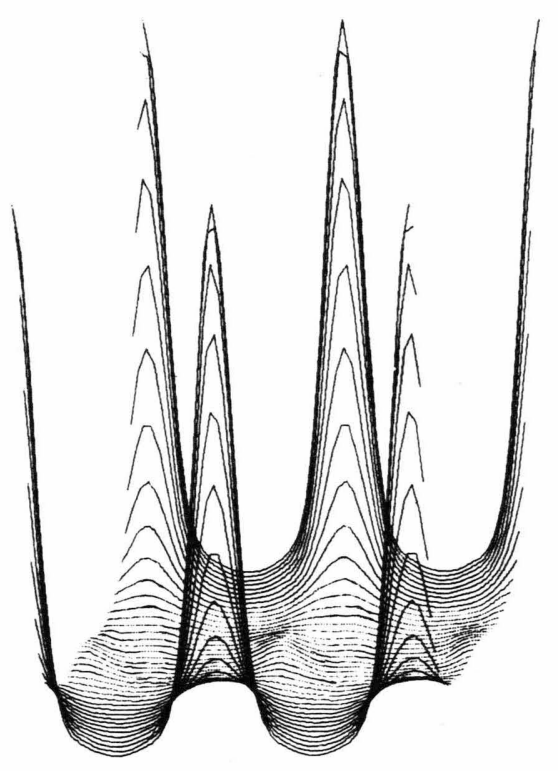

b)

crystal and they are repelled from the rows of positively charged nuclei. This effect is more pronounced for $500 \mathrm{keV}$ positrons. Positrons, travelling along the [110] axis, are concentrated mainly in one wave field (Fig. 9a). Since the positrons are concentrated between the atoms at regions of least elastic interaction with the lattice, this one Bloch wave describes a stable situation. All other wave fields are very weak. Electrons, however, travel in many wave fields through the crystal. The three strongest ones are plotted in Figures $9 \mathrm{~b}-\mathrm{d}$. Due to the particle picture the electrons are
A deeper understanding we will find by looking at the Bloch waves for positrons, shown in Figure 8. In wavefield 1 the positrons are concentrated between the atoms and in the other two wave fields one can see how the positrons avoid the atoms. Because of their positive charge they prefer regions of negative charge in the

Fig. 9. (a) The strong positron Bloch wave at $G=0$ and $H=0 ;(\mathrm{b}-\mathrm{d})$ the strongest electron Bloch waves at $G=0$ and $H=0$
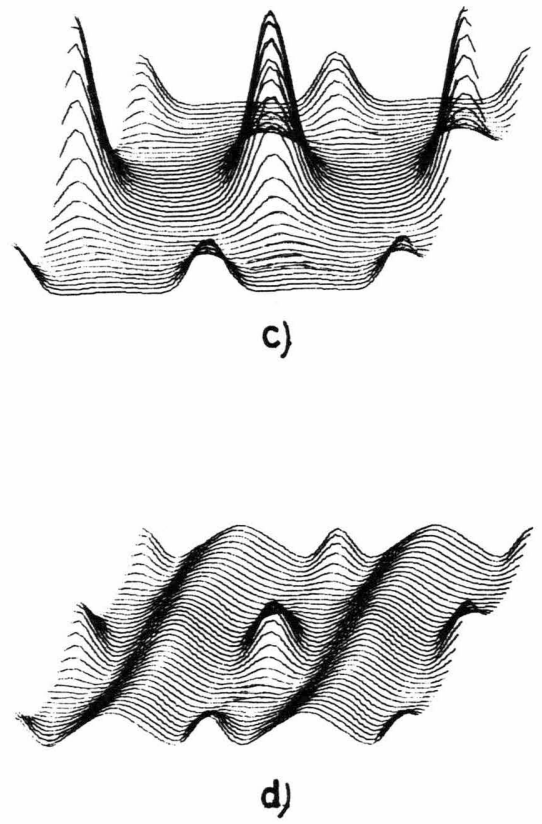

attracted by the rows of the positive atoms, suffering strong elastic and inelastic interaction with the crystal. These electrons we find in wave field number one of the wave mechanical picture. The electrons scattered away from the rows of atoms one finds in the other wave fields. It is interesting to see that wave field three is weaker for this orientation than for the orientation $G=0, H=2$ shown in Figure 2. Furthermore this wave field has density maxima at the positions of the atoms and consequently it will be more strongly absorbed than in the case of $G=0$ and $H=2$. The dif- 
ference of the absorption coefficients, leading to the Borrmann effect, will be smaller and so the anomalous transmission will be less for this orientation in agreement with observations in zone axes Kikuchi-pattern shown by Uyeda and Nonoyama ${ }^{17}$ and recently by Fujimoto et. al. ${ }^{18}$.
It is a great pleasure for me to thank Dr. KamBE for many discussions and also Dr. WAGENFELD and Prof. Fujiмото for suggesting the positron calculations.

\title{
M1 Transitions in the Reaction ${ }^{6} \mathrm{Li}\left(\gamma,{ }^{2} \mathrm{H}\right){ }^{4} \mathrm{He}$
}

\author{
K. H. PASSLER \\ Institut für Theoretische Physik der Justus-Liebig-Universität Gießen, Germany \\ (Z. Naturforsch. 28a, 8-11 [1973]; received 10 October 1972)
}

\begin{abstract}
It is shown that the admixture of a $\mathrm{D}$-state in the wave function for the relative motion of the $\alpha$-particle and the deuteron in the nucleus ${ }^{6} \mathrm{Li}$ leads to $\mathrm{M} 1$ transitions. Their mixture with E2 transitions does not lead to an asymmetry of the differential cross section of the reaction ${ }^{6} \mathrm{Li}\left(\gamma,{ }^{2} \mathrm{H}\right)^{4} \mathrm{He}$ about $90^{\circ}$, and they are negligibly small.
\end{abstract}

According to a selection rule ${ }^{1}$ which follows from the charge symmetry of nuclear forces, E1 transitions in self-conjugate nuclei without change of the total isospin quantum number are forbidden. However it was shown ${ }^{2}$ that in the derivation of this rule an assumption was made which is not valid in the cluster model. This assumption reads that the charge-to-mass ratios of all groups of nucleons in a nucleus are equal.

The effects of clustering are expected to be relatively strong in the nucleus ${ }^{6} \mathrm{Li}$. Calculations show ${ }^{3}$ that the binding energies of the ${ }^{2} \mathrm{H}$ and ${ }^{4} \mathrm{He}$ clusters are very close to the binding energies of the corresponding free particles. So it is a good approximation to use the masses of the free particles for the masses of these clusters.

A possible violation of this isospin selection rule can be tested in the reaction

$$
{ }^{6} \mathrm{Li}\left(\gamma,{ }^{2} \mathrm{H}\right){ }^{4} \mathrm{He} \text {. }
$$

Reprint requests to Dipl.-Phys. Karl-Heinz Passler, Institut für Theoretische Physik, D-6300 Gießen, Leihgesterner Weg 108.

1 E. K. Warburton and J. Weneser, in: Isospin in Nuclear Physics, edited by D. H. Wilkinson, North Holland Publishing Company, Amsterdam 1969, p. 215.
First we will assume that in the ground state of ${ }^{6} \mathrm{Li}$ the two clusters are in a relative $\mathrm{S}$-state. Then there are no M1 transitions. Therefore, if the selection rule is valid only E2 transitions can contribute, so the differential cross section is predicted to be symmetrical about $90^{\circ}$. On the other hand, if clustering effects are important, the selection rule is violated and E1 transitions can occour. The mixture of these $\mathrm{E} 1$ transitions with the E2 transitions then would cause an asymmetry of the differential cross section.

However, the nonvanishing quadrupole moment of the nucleus ${ }^{6} \mathrm{Li}$ indicates that there may be an admixture of a $\mathrm{D}$-state in the relative motion. This would allow M1 transitions, which then would lead to interference terms with the $\mathrm{E} 2$ transitions. Thus, even if the isospin selection rule is not violated, the angular distribution of the reaction products may not be that of a pure $\mathrm{E} 2$ transition.

- V. I. Mamasakhlisov and T. S. Macharadze, Sov. J. Nucl. Phys. 9, 198 [1969].

3 E. W. SCHMid, Y. C. TANG, and K. WILDERMUth, Phys. Letters 7, 263 [1963].

4 J. L. Gammel, B. J. Hill, and R. M. Thaler, Phys. Rev. 119, 267 [1960]. 